\title{
Bayesian Inference for Materials Physics from STEM Data: The Probability Distribution of Physical Parameters from Ferroelectric Domain Wall Observations
}

Christopher Nelson ${ }^{1}$, Rama Vasudevan ${ }^{2}$, Xiaohang Zhang ${ }^{3}$, Maxim Ziatdinov ${ }^{4}$, Eugen Eliseev ${ }^{5}$, Ichiro Takeuchi $^{3}$, Anna Morozovska ${ }^{6}$ and Sergei Kalinin ${ }^{7}$

${ }^{1}$ Oak Ridge National Laboratory, Oak Ridge, Tennessee, United States, ${ }^{2}$ Oak Ridge National Laboratory, United States, ${ }^{3}$ University of Maryland, Maryland, United States, ${ }^{4}$ Computational Sciences and Engineering Division, Oak Ridge National Laboratory, Oak Ridge, Tennessee, United States, ${ }^{5}$ Institute for Problems of Materials Science, National Academy of Sciences of Ukraine, United States, ${ }^{6}$ Institute of Physics, National Academy of Sciences of Ukraine, United States, ${ }^{7}$ Center for Nanophase Materials Sciences, Oak Ridge National Laboratory, Oak Ridge, Tennessee, United States

Bayesian inference provides the probability of a model / hypothesis given a set of experimental observations, a powerful statistical technique rising in popularity with the availability of corresponding computational and statistical tools. The utility of this method towards analyzing scientific data as from (S)TEM is that in many cases the necessary models/hypotheses are available a-priori, being derived from known underlying physics. Moreover, the outcome goes beyond a best-fit solution allowing evaluation of uncertainty from the parameter probability distributions. The aim of this work is to compute the posterior distributions of the parameters for physics models, conditioned on experimental STEM data, and evaluate the probabilities for competing models. For a model system we develop the Bayesian inference framework to evaluate observations of a twinning boundary against models for first vs. second-order ferroelectric phase transitions.

For the inputs we employ weakly informative priors and Bayesian regression models for 1-D ferroelectric domain walls using a Ginzburg-Landau-Devonshire expansion. The competing models correspond to (\#1) $2^{\text {nd }}$ order, (\#2) 1st order, and (\#3) $1^{\text {st }}$ order with rotation (i.e. two component). Experimental observations are extracted from a HAADF-STEM dataset (Figure 1) by taking each atom-row transecting a 2-D ferroelectric domain wall as an independent observation. For the polarization term in the GLD equations we use a proxy measurement of the local lattice-coupling via the phase offset of the local A- and B- site sublattices [1].

The Bayesian analysis is applied to subregions exhibiting segments of $109^{\circ}$ (Fig 1c) and $180^{\circ}$ (Fig 1d) domain walls. Select results for the $109^{\circ}$ case are shown in Figure 2 consisting of plots of the three models (a), posterior probabilities of the physical model parameters (b), and joint probability distributions (c). The outcome in both cases is the $1^{\text {st }}$ order models $(2 \& 3)$ resolve negligible values for the parameters that distinguish them from the simpler $2^{\text {nd }}$ order model (1) giving them nearly identical profiles (see Fig 1a) and indicating Model 1 to be the most probable solution. However, the parameter probabilities, and thus uncertainties, can be explicitly observed in their posterior distributions (for example in Fig 2b,c). A comparison of the three models using the widely applicable information criteria (WAIC) [2] gives only a 5:2 and 5:3 ratio of weights for Model 1 against 2 and 3 respectively. Analysis using synthetic data reveals this uncertainty is largely driven by the relatively low spatial sampling (atomic sites) relative to the short correlation lengths of ferroelectric domain walls in $\mathrm{BiFeO}_{3}$. 
a

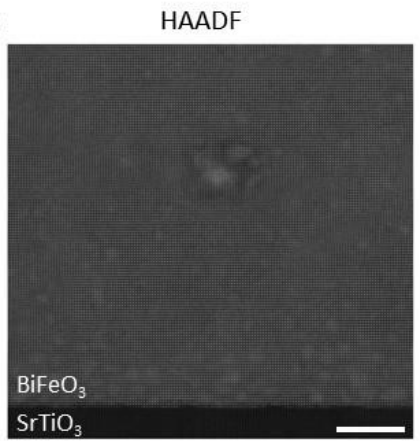

d

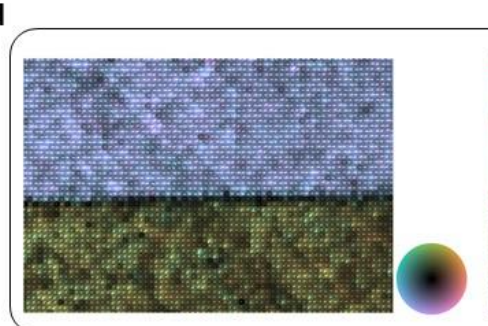

b

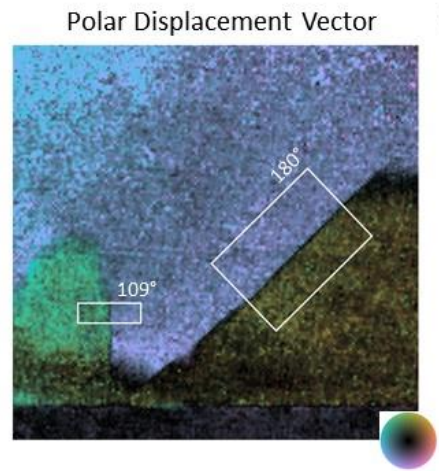

$180^{\circ}$ Domain Wall
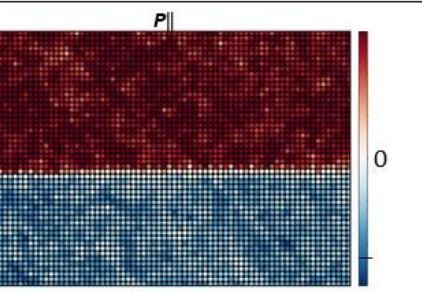

c
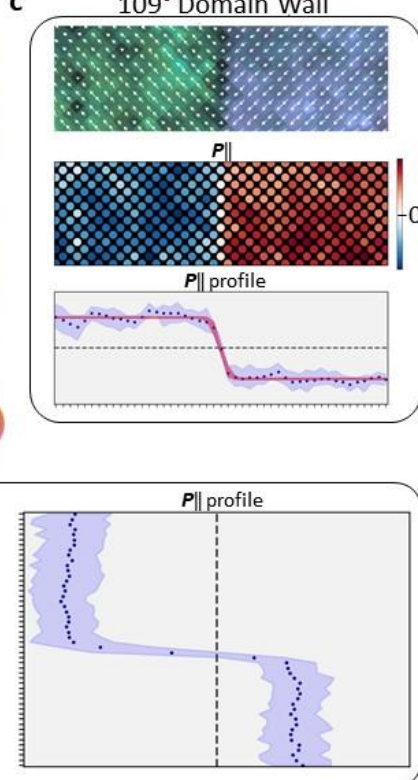

Figure 1. Figure 1. Parameterized HRSTEM data (a) HAADF-STEM of BiFeO3 film on SrTiO3 substrate, scalebar 10nm. (b) Cation polar displacement vector (P) map, white rectangles depict subregion datasets bisecting $109^{\circ}$ and $180^{\circ}$ domain walls. (c,d) Bayesian analysis of $109^{\circ}$ and $180^{\circ}$ domain wall subregions, respectively. Each shows the corresponding displacement vector map, a colorized scatter plot of $\mathrm{P}$ component parallel ([001]pc) to the domain wall, and the domain wall profile along the normal axis. In the latter, the red band corresponds to the $90 \%$ highest posterior density interval for the Bayesian analysis Model \#2, the blue band is the $90 \%$ data bounds, and the datapoints are mean values. 

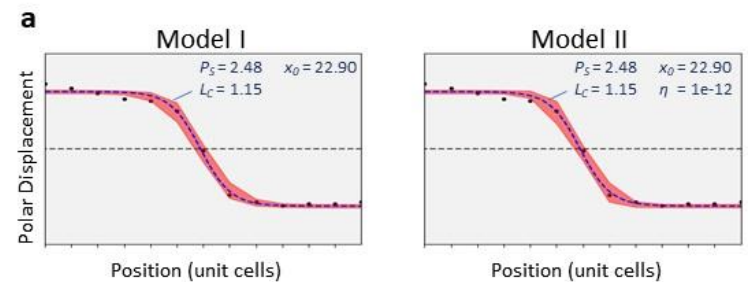

Position (unit cells)

C

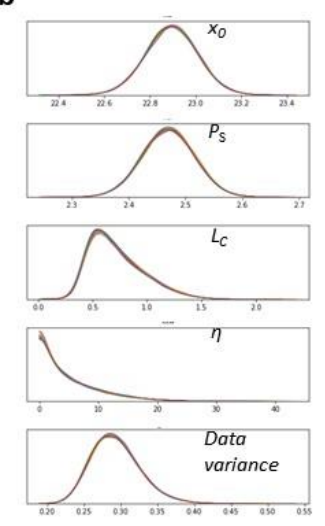

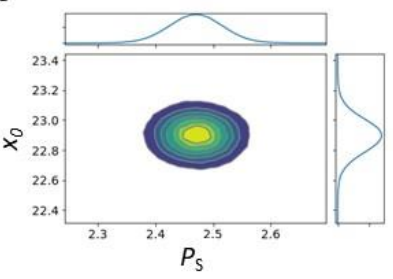

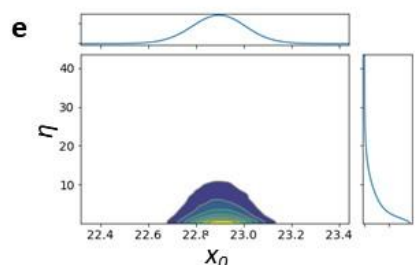

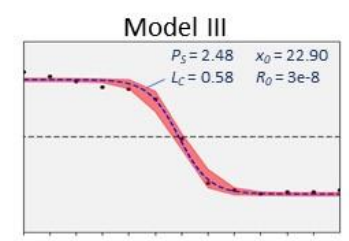

Position (unit cells)

d

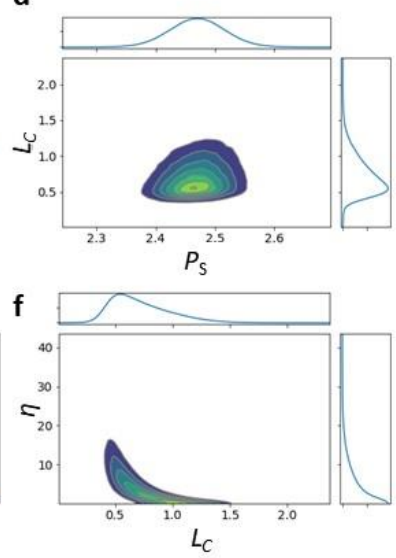

Figure 2. Figure 2. $109^{\circ}$ Domain wall: GLD models and posterior probability densities. (a) The $90 \%$ highest posterior density interval for the Bayesian analysis (red band) overlaid on experimental mean values (data points). Dashed blue line is a least square fit. (b) Posterior probability densities (PPD) for the Model 2 parameters: wall position, saturation polarization, correlation length, eta, and the data variance. (c-f) are selected 2D joint probability densities for different parameter combinations.

\section{References}

[1] C. L. Jia et al., Nature Materials 6 (2007), p. 64.

[2] Y. Liu et al., J. Mater. Res. 32 (2017), p. 847.

[3] This work was supported by the U.S Department of Energy, Office of Science, Basic Energy Sciences, Materials Sciences and Engineering Division. 